1 Universidade Federal da Bahia(UFBA), Instituto de Saúde Coletiva (ISC) Salvador (BA), Brasil. elzojr@hotmail.com

2 Agência Nacional de Saúde Suplementar (ANS)

- Rio de Janeiro (RJ), Brasil. julianaenfbs@hotmail.com

3 Governo do Estado do Ceará, Secretaria da Saúde do Estado do Ceará Fortaleza (CE), Brasil. betamor_betamor@hotmail. com

4 Universidade Estadual do Ceará (UECE), Centro de Ciências da Saúde, Departamento de Enfermagem - Fortaleza (CE), Brasil.

anapatricia.morais@uece.br

5 Universidade Estadual do Ceará (UECE), Programa de Pós-Graduação em Saúde Coletiva - Fortaleza (CE), Brasil.

marcelo.gurgel@uece.br

\section{Análise da produção científica sobre avaliação, no contexto da saúde da família, em periódicos brasileiros}

\author{
Analysis of scientific production on evaluation, in the context of \\ family health, in Brazilian journals
}

\author{
Elzo Pereira Pinto Junior'1, Juliana Lucena de Miranda Cavalcante², Roberta Alves Sousa ${ }^{3}$, Ana \\ Patrícia Pereira Morais ${ }^{4}$, Marcelo Gurgel Carlos da Silva ${ }^{\mathbf{5}}$
}

RESUMO Este estudo buscou analisar a bibliografia relacionada à avaliação no contexto da Estratégia Saúde da Família, no Brasil, em periódicos de saúde coletiva, de 2003 a 2012. A análise concentrou-se na identificação de 'autores', 'ano de publicação', 'instituição do primeiro autor', 'periódico', 'local', 'método utilizado', 'marco conceitual' e 'grupo de estudos'. O corpus documental contou com 20 artigos completos, que demonstraram intensificação na produção de estudos na área da avaliação. Nas abordagens metodológicas prevaleceram estudos quantitativos, com diversidade de marcos conceituais. Portanto, mensurações sobre o impacto de ações da ESF são imprescindíveis na melhoria da qualidade da avaliação e da própria ESF, no contexto da saúde coletiva.

PALAVRAS-CHAVE Avaliação em saúde; Estratégia Saúde da Família; Atenção Primária à Saúde; Indicadores de produção científica.

ABSTRACT This study aims to analyze the bibliography related to the assessment in the context of the Family Health Strategy, in Brazil, in Collective Health journals, from 2003 to 2012. The analysis has concentrated on the identification of authors, 'year of publication', 'institution of the first author', 'place', 'used method', 'conceptual landmark' and 'group of studies'. The documentary Corpus counted on 20 complete articles that have demonstrated intensification in the production of studies in the evaluation area. In the methodological approaches quantitative studies had prevailed, with diversity of conceptual landmarks. Therefore, measurements about the impact of the ESF actions are essential in the improvement of the quality of evaluation and the $\mathrm{ESF}$ itself, in the collective health context.

KEYWORDS Health evaluation; Family Health Strategy; Primary Health Care; Scientific publication indicators. 


\section{Introdução}

A atividade avaliativa é tão antiga quanto a própria humanidade, haja visto que a emissão de juízo de valor é inerente às ações humanas. A avaliação tem-se constituído como uma área com muitos conceitos, portanto, polissêmica e com diversas metodologias, que se modificam e se adaptam a determinados setores sociais e a diferentes contextos históricos. Uma proposta de definição de avaliação descreve-a como a aplicação de um julgamento de valor a uma intervenção, através de um dispositivo que fornece informações científicas legítimas, fazendo com que os envolvidos possam tomar suas posições e decisões (CONTANDRIOPOULOS; CHAMPAGNE, 2006).

No Brasil, a avaliação em saúde tem se constituído como uma política, na medida em que as reformas e as ações na saúde passam a exigir maiores competências gerenciais, articulações e produção de informação. Nesse cenário de demandas por resultados, monitoramentos e avaliações, tornou-se necessário instituir a avaliação com o propósito fundamental de dar amparo aos processos decisórios, subsidiar a identificação de problemas, reorientar ações e serviços, avaliar a incorporação de novas práticas e aferir o impacto das ações implementadas pelos serviços de saúde (BRASIL, 2005).

A Atenção Primária à Saúde (APS), como primeiro nível de atenção à saúde do Sistema Único de Saúde (SUS), com maior capilaridade de ações destinadas à maioria da população, passa a ser o principal espaço para a aplicação de processos avaliativos. Neste contexto, a Estratégia Saúde da Família (ESF), definida como o modelo de atenção capaz de fortalecer e organizar a APS, revela-se como campo ideário de avaliações em saúde.

A avaliação da ESF, apesar de necessária, pode ser considerada uma tarefa difícil de ser implementada. Fatores como a extensão territorial e a diversidade cultural do País, entre outros aspectos, contribuem para a dificuldade de institucionalização do processo avaliativo nesses serviços. Assim, a avaliação realizada deverá levar em consideração o fato de essa estratégia estar em contínua implementação, exigindo a inserção de novos critérios, além de reconhecer os possíveis vieses existentes (LENTSCK; KLUTHCOVSKY; KLUTHCOVSKY, 2010).

Após 19 anos de existência e uma significativa abrangência nacional, espera-se que haja avaliações das ações realizadas pelas ESF, estimuladas tanto por órgãos governamentais quanto por instituições de ensino e pesquisa. Assim, fazem-se necessários estudos que busquem realizar um diagnóstico das principais pesquisas relacionadas aos programas e estratégias desenvolvidas, que possam dar margem a uma compreensão de como estão sendo realizadas essas avaliações e sob que abordagem elas estão se dando.

Portanto, buscamos, neste estudo, analisar a produção bibliográfica relacionada à avaliação no contexto da ESF, no Brasil, em periódicos selecionados da área da saúde coletiva.

\section{Metodologia}

Realizou-se uma análise bibliográfica acerca das pesquisas que envolvessem a avaliação em saúde no contexto da ESF. A pesquisa bibliográfica se caracteriza como uma fonte importante de informações, contribuindo para a construção do saber, como a atividade intelectual e o conhecimento cultural. Ocupando lugar de destaque entre as demais, ela é um conjunto de conhecimentos das mais variadas obras, que proporciona ao leitor ferramentas para a elaboração de pesquisas futuras (FACHIN, 2006).

Como critérios de inclusão para compor esse estudo bibliográfico, optou-se por considerar como relevantes apenas os artigos publicados em periódicos nacionais de boa qualidade na área de saúde coletiva. Para atender a esse critério, os autores consultaram o WebQualis ${ }^{\mathbf{1}}$, onde puderam listar os periódicos brasileiros com avaliação situada
1 Disponível em: <qualis capes.gov.br/webqualis>. 
2 Em inglês: Primary Care Assessment Tool (PCATool), instrumento de avaliação da APS. nas categorias de maior qualificação para os periódicos desta área ( $\mathrm{A} 1, \mathrm{~A} 2 \mathrm{e} \mathrm{B1})$. Além disso, optou-se, ainda, por limitar o intervalo de tempo de publicação nos últimos dez anos, realizando busca entre os anos 2003-2012, por considerar esse período uma fase de consolidação e qualificação das pesquisas em avaliação em saúde no Brasil, especialmente no contexto da ESF. Para o levantamento dos periódicos, foi acessado o WebQualis, em 03 de fevereiro de 2013, para a identificação daqueles que apresentam a qualificação especificada.

Posteriormente, foi feita uma busca dos artigos nas bases de dados do Centro LatinoAmericano e do Caribe de Informação em Ciências da Saúde (Bireme) e da Literatura Latino-Americana e do Caribe em Ciências da Saúde (Lilacs). A busca utilizou como descritores os termos 'Avaliação', 'Avaliação em Saúde', 'Programa de Saúde da Família' e 'Atenção Básica', todos componentes dos Descritores em Ciências da Saúde (DeCS), da Bireme. Como estratégia de busca, foram realizadas as seguintes combinações dos descritores supracitados: ['Avaliação em Saúde' AND 'Programa de Saúde da Família'], ['Avaliação em Saúde’ AND ‘Atenção Básica’], ['Avaliação' AND 'Programa de Saúde da Família'] e ['Avaliação' AND 'Atenção Básica']. Apesar de a atual nomenclatura adotar o termo Estratégia Saúde da Família, a busca foi realizada com o termo Programa Saúde da Família, a fim de garantir padronização ao descritor presente no DeCS.

Procedeu-se a busca dos textos completos desses artigos, e os que não estavam disponíveis online foram excluídos do estudo. De todos os artigos, antes da seleção, foram analisados os resumos, os títulos e a metodologia, com o intuito de perceber se havia, de certo, alguma correlação com o tema abordado. Nos casos em que o resumo não deixava claro o objetivo da pesquisa, esse dado foi buscado pela análise do artigo completo.

Após a coleta das referências, foi feita a verificação de duplicidade para que se procedesse à leitura dos resumos dos artigos selecionados nas quatro estratégias de busca adotadas. Os resumos foram lidos e optou-se por excluir aqueles cuja abordagem não se vinculava à avaliação no contexto da ESF. Não se fez distinção entre os textos que abordavam métodos quantitativos, qualitativos ou mistos, sendo todos elegíveis. Também foram considerados passíveis de serem selecionados textos com discussões teórico-metodológicas ou que apontassem novas abordagens nessa temática.

Após essa segunda etapa de leitura, ainda foram excluídos alguns textos, pois foi verificado que os seus objetos de estudo não poderiam responder aos objetivos desta pesquisa. As quatro estratégias de busca localizaram 386 artigos. Após a aplicação dos critérios de inclusão e exclusão, foram selecionados 236 resumos para leitura. A seleção desses resumos apontou a necessidade de aprofundamento em 50 textos, que foram lidos em sua íntegra, restando 20 artigos para compor o corpus desta pesquisa.

Os artigos selecionados para compor o corpo dos resultados foram analisados quanto aos seguintes aspectos: 'autores', 'ano de publicação do estudo', 'instituição do primeiro autor', 'periódico onde foi publicado', 'local de realização do estudo', 'método utilizado do estudo', 'marco conceitual adotado para trabalhar o conceito de avaliação' e 'grupo de estudos de avaliação'. Em relação ao 'método utilizado', consideraram-se os seguintes tipos: quantitativo, qualitativo, misto (triangulação de métodos) e teórico-metodológico-reflexivo. No aspecto 'grupo de estudos de avaliação', optou-se por descrever a iniciativa por trás do estudo, ou seja, atribuir-lhe um fio condutor, como o Programa de Expansão da Estratégia Saúde da Família (Proesf) e seus estudos de linha de base, ou ainda os estudos em Internações por Condições Sensíveis à Atenção Primária (ICSAP), além das pesquisas que abordam os atributos da APS com a aplicação do PCATool ${ }^{2}$, entre outros. 
Para sistematizar a análise dessas variáveis foram construídos dois quadros de caracterização, descritos nos resultados como quadro 1 e quadro 2. Os dados foram organizados em planilha do programa Excel, sendo realizada análise por meio das frequências absoluta e relativa.

\begin{tabular}{|c|c|c|c|}
\hline Primeiro Autor & Ano & Instituição do Primeiro Autor & Periódico \\
\hline Campos & 2005 & $\begin{array}{l}\text { Universidade Federal do } \\
\text { Rio de Janeiro (UFRJ) - RJ }\end{array}$ & Revista Brasileira de Saúde Materno Infantil \\
\hline Facchini & 2006 & $\begin{array}{l}\text { Universidade Federal de } \\
\text { Pelotas (UFPel) - RS }\end{array}$ & Ciência \& Saúde Coletiva \\
\hline Ibañez & 2006 & $\begin{array}{l}\text { Faculdade de Ciências Médicas da Santa } \\
\text { Casa de São Paulo (FCMSCSP) - SP }\end{array}$ & Ciência \& Saúde Coletiva \\
\hline Szwarcwald & 2006 & Fundação Oswaldo Cruz (Fiocruz) - RJ & Ciência \& Saúde Coletiva \\
\hline Scochi & 2008 & $\begin{array}{l}\text { Universidade Estadual } \\
\text { de Maringá (UEM) - PR }\end{array}$ & Cadernos de Saúde Pública \\
\hline Camargo Jr. & 2008 & $\begin{array}{l}\text { Universidade do Estado do Rio de Janeiro } \\
\text { (Uerj) - RJ }\end{array}$ & Cadernos de Saúde Pública \\
\hline Elias & 2008 & $\begin{array}{l}\text { Universidade do Sul de } \\
\text { Santa Catarina (Unisul) - SC }\end{array}$ & Revista Brasileira de Epidemiologia \\
\hline Tanaka & 2008 & Universidade de São Paulo (USP) - SP & Revista Brasileira de Saúde Materno Infantil \\
\hline Medina & 2009 & $\begin{array}{l}\text { Universidade Federal da } \\
\text { Bahia (UFBA) - BA }\end{array}$ & Cadernos de Saúde Pública \\
\hline Gomes & 2009 & $\begin{array}{l}\text { Universidade Federal de } \\
\text { Viçosa (UFV) - MG }\end{array}$ & Ciência \& Saúde Coletiva \\
\hline Fernandes & 2009 & $\begin{array}{l}\text { Universidade Estadual de Montes Claros } \\
\text { (Unimontes) - MG }\end{array}$ & Revista de Saúde Pública \\
\hline Silva & 2010 & $\begin{array}{l}\text { Universidade Estadual de Montes Claros } \\
\text { (Unimontes)- MG }\end{array}$ & Cadernos de Saúde Pública \\
\hline Leão & 2011 & $\begin{array}{l}\text { Universidade Estadual de Montes Claros } \\
\text { (Unimontes)- MG }\end{array}$ & Ciência \& Saúde Coletiva \\
\hline Rehem & 2011 & Universidade de São Paulo (USP) - SP & Ciência \& Saúde Coletiva \\
\hline Uchoa & 2011 & $\begin{array}{l}\text { Universidade Federal do Rio Grande do Norte } \\
\text { (UFRN) - RN }\end{array}$ & Physis \\
\hline Caldeira & 2011 & $\begin{array}{l}\text { Universidade Estadual de Montes Claros } \\
\text { (Unimontes) - MG }\end{array}$ & Revista Brasileira de Saúde Materno Infantil \\
\hline Sala & 2011 & $\begin{array}{l}\text { Secretaria de Estado de } \\
\text { Saúde (SES) - SP }\end{array}$ & Saúde e Sociedade \\
\hline Tanaka & 2011 & USP - SP & Saúde e Sociedade \\
\hline Cardoso & 2012 & $\begin{array}{l}\text { Universidade Federal da } \\
\text { Bahia (UFBA) - BA }\end{array}$ & Cadernos de Saúde Pública \\
\hline Boing & 2012 & $\begin{array}{l}\text { Universidade Federal de } \\
\text { Santa Catarina (UFSC) - SC }\end{array}$ & Revista de Saúde Pública \\
\hline
\end{tabular}

Fonte: Elaboração própria 


\begin{tabular}{|c|c|c|c|c|}
\hline Autores & Local do Estudo & Tipo de Estudo & Marco Conceitual Adotado & Estratégia de Pesquisa \\
\hline Silva & Montes Claros (MG) & Transversal, Descritivo & $\begin{array}{l}\text { Avaliação para a Melhoria da } \\
\text { Qualidade da ESF }\end{array}$ & $\begin{array}{l}\text { Avaliação para Melhoria da } \\
\text { Qualidade (AMQ) }\end{array}$ \\
\hline Sala & São Paulo (SP) & Quantitativo, Transversal & Starfield - Atributos da APS & Atributos APS \\
\hline Leão & Montes Claros (MG) & $\begin{array}{l}\text { Transversal, Descritivo, } \\
\text { Analítico }\end{array}$ & Starfiel - Atributos da APS & Atributos APS \\
\hline Caldeira & Montes Claros (MG) & Transversal, Analítico & CSAP e Efetividade da ESF & ICSAP \\
\hline Rehem & Estado de São Paulo & Descritivo, Ecológico & CSAP e Efetividade da ESF & ICSAP \\
\hline Boing & Florianópolis (SC) & Quantitativo & $\begin{array}{l}\text { ICSAP e Efetividade da atenção } \\
\text { primária }\end{array}$ & ICSAP \\
\hline Fernandes & Montes Claros (MG) & Quantitativo & $\begin{array}{l}\text { ICSAP e Efetividade da atenção } \\
\text { primária }\end{array}$ & ICSAP \\
\hline Gomes & Paula Cândido (MG) & Quantitativa & ICSAP & ICSAP \\
\hline Elias & Estado de Santa Catarina & Quantitativo & $\begin{array}{l}\text { ICSAP e Efetividade da atenção } \\
\text { primária }\end{array}$ & ICSAP \\
\hline Medina & Estado da Bahia & Triangulação de Métodos & - & Não se aplica \\
\hline Tanaka & Não se aplica & Reflexivo-Teórico & Donabedian & Não se aplica \\
\hline Tanaka & São Paulo (SP) & Quantitativo & Donabedian e Kessner & Não se aplica \\
\hline Campos & Não se aplica & Reflexivo-Teórico & Donabedian & Não se aplica \\
\hline Cardoso & Salvador (BA) & Quantitativo & $\begin{array}{l}\text { Soberón (cobertura real e } \\
\text { potencial) }\end{array}$ & Não se aplica \\
\hline Camargo Jr. & Estado do Rio de Janeiro & Triangulação de Métodos & Contradiopoulus e Kessner & Não se aplica \\
\hline Uchoa & Estado do Rio Grande do Norte & Qualitativo & Donabedian & Não se aplica \\
\hline Ibañez & $\begin{array}{l}\text { Municípios do Estado de São } \\
\text { Paulo }\end{array}$ & Quantitativo & Starfield - Atributos da APS & PCATool \\
\hline Scochi & Municípios do Estado do Paraná & $\begin{array}{l}\text { Avaliação de Quarta } \\
\text { Geração e Pesquisa - } \\
\text { Ação }\end{array}$ & $\begin{array}{l}\text { Política de Avaliação da Atenção } \\
\text { Básica - Ministério da Saúde }\end{array}$ & $\begin{array}{l}\text { Projeto de Expansão e } \\
\text { Consolidação Saúde da Família } \\
\text { (Proesf) }\end{array}$ \\
\hline Facchini & $\begin{array}{l}\text { Estados de Alagoas, do Pará, do } \\
\text { Piauí, do Rio Grande do Norte, } \\
\text { de Pernambuco, do Rio Grande } \\
\text { do Sul e de Santa Catarina }\end{array}$ & Quantitativo & - & $\begin{array}{l}\text { Projeto de Expansão e } \\
\text { Consolidação Saúde da Família } \\
\text { (Proesf) }\end{array}$ \\
\hline Szwarcwald & Estado do Rio de Janeiro & Quantitativo & Pesquisa Mundial da Saúde & $\begin{array}{l}\text { Projeto de Expansão e } \\
\text { Consolidação Saúde da Família } \\
\text { (Proesf) }\end{array}$ \\
\hline
\end{tabular}

\section{Resultados e discussão}

\section{Descrição dos artigos}

Foram incluídos, no corpus documental desta pesquisa, 20 artigos. A partir da análise do quadro 1 é possível verificar as produções no que se referem às datas de publicações, às instituições envolvidas na pesquisa e aos periódicos que publicaram tais textos.

Pode-se perceber uma intensificação na produção dos estudos em avaliação em saúde no contexto da ESF nos últimos quatro anos, tendo em vista que 16 (80\%) trabalhos foram publicados entre 2008 e 2012. Lentsck, Kluthcovsky e Kluthcovsky (2010), em artigo publicado na revista Ciência \& 
Saúde Coletiva - que, de forma semelhante, fez um levantamento bibliográfico sobre a avaliação do Programa Saúde Família (PSF), em período que antecede a este estudo -, observaram uma concentração das publicações nos anos de 2005 e 2006. Tal estudo identificou ainda que os primeiros trabalhos relacionados à temática da avaliação do PSF datam de 2001, ou seja, sete anos depois da sua implementação. Um lapso de tempo importante que foi desperdiçado, demonstrando, com isso, tanto o caráter inicial e imaturo do programa como a dificuldade na institucionalização do processo de avaliação (LENTSCK; KLUTHCOVSKY; KLUTHCOVSKY, 2010).

Apesar de alguns estudos fazerem referência à evolução das análises do desenvolvimento do PSF ao longo dos anos (HENRIQUE; CALVo, 2008), na sua grande maioria estão voltados para áreas de atendimento específicas, como as relacionadas à saúde da mulher (MOURA; HOLANDA; RODRIGUES, 2003) e à saúde da criança, além de delimitados a espaços geográficos bem definidos (GOMES; SILVA, 2011).

Em relação às instituições de ensino superior e aos centros de pesquisas responsáveis pela condução dos estudos nessa temática, fica claro o predomínio da produção desses conhecimentos na região Sudeste do Brasil, já que, de todos os trabalhos que fazem parte desta análise, 14 (70\%) são produzidos por instituições situadas nos estados de Minas Gerais, São Paulo ou Rio de Janeiro.

Esse fato difere dos resultados encontrados por Lentsck, Kluthcovsky e Kluthcovsky (2010), que observaram uma prevalência de estudos relacionados à avaliação, tanto na região Nordeste quanto na região Sudeste, e uma carência desse tipo de estudo nas regiões Norte e Centro-Oeste. Esta diferença pode fazer sentido, visto que a implantação do PSF inicia-se na região Nordeste e que, na época, existiam muitas instituições de ensino e pesquisas envolvidas nesse processo. Atualmente, com a expansão da saúde da família em todo o território nacional, destacam-se, no cenário da produção científica, as instituições com cursos de Pós-Graduação mais bem conceituados e estruturados, como os encontrados no Sul e no Sudeste do Brasil.

$\mathrm{Na}$ análise dos periódicos, o Cadernos de Saúde Pública e o Ciência \& Saúde Coletiva foram responsáveis por 11 (55\%) artigos, o que pode indicar uma política editorial compatível com a produção e a divulgação dos estudos com a temática da avaliação na ESF. Nesse aspecto, há de se considerar que tais periódicos têm vinculação com instituições de ensino e pesquisa também situadas em localidades da região Sudeste do Brasil, centro da produção de conhecimento no País, devido aos volumes de pesquisadores e recursos empregados nesse setor.

\section{Abordagens metodológicas dos estudos em avaliação em saúde no contexto da ESF}

\section{TIPOS DE ESTUDO}

Com relação à tipologia dos estudos, constatou-se um predomínio do método quantitativo, seguido pela triangulação de métodos e estudos téorico-reflexivos acerca do processo de avaliação (quadro 2). O resultado desta pesquisa diverge do encontrado por Lentsck, Kluthcovsky e Kluthcovsky (2010), que observou um equilíbrio entre os métodos utilizados, em que $50 \%$ dos artigos analisados se caracterizavam por ser de abordagem metodológica qualitativa e apenas $31,8 \%$, quantitativa.

Nesse sentido, torna-se importante discorrer sobre os tipos de abordagens metodológicas identificadas no escopo dos 20 artigos, de modo a facilitar a compreensão em relação à abordagem conceitual e às metodologias das avaliações implementadas.

Os estudos quantitativos se constituem essencialmente a partir dos princípios do paradigma positivista e buscam a explicação do comportamento dos objetos e suas variáveis, por meio do estabelecimento matemático da relação entre os itens que se pretende 
investigar. A seleção de sujeitos nesse tipo de estudo envolve aleatorização e amostragem, e sua análise é feita com base em conhecimentos de estatística, para que se alcancem as conclusões (TURATO, 2005).

Outra abordagem de estudo é a que envolve uma tipologia qualitativa. De acordo com Minayo (1993), uma pesquisa com abordagem qualitativa se preocupa em desvendar os sentidos e significados dos processos, bem como compreender a complexidade do comportamento dos seres humanos. Não pretende, desse modo, quantificar casos ou utilizar-se de métodos estatísticos para tratar resultados. Esse tipo de pesquisa permite que se estude uma ampla variedade de fenômenos sociais, possibilitando o aprofundamento na compreensão de comportamentos, percepções e atitudes humanas diante de determinada realidade social (MARCONI; LAKATOS, 2004).

Buscando uma superação da dicotomia entre o método quantitativo e o qualitativo, ainda mais se tratando de pesquisas avaliativas, Minayo, Assis e Souza (2005) desenvolveram a alternativa conhecida como 'triangulação de métodos', que pode ser compreendida como uma dinâmica de investigação que integra a análise sob uma ótica ampliada, considerando estruturas, processos e resultados, a uma compreensão de relações e atores envolvidos.

As abordagens metodológicas identificadas por este estudo (quadro 2) demonstram uma consequência dos caminhos traçados pela avaliação em saúde em nosso país. Resguardada a perspectiva do estilo autoral, as publicações sobre avaliação realizadas junto à ESF têm a finalidade de medir estruturas, indicadores, resultados e impactos, amplitude e cobertura de ações, e grau de satisfação de usuário, de forma a responder sobre a qualidade e a efetividade dos serviços e ações ofertados. Os aspectos elencados suscitam o uso de métodos lógico-estatísticos pela natureza do objeto da avaliação, mas também no atendimento ao objetivo da avaliação. Pois, no campo da avaliação em saúde, três questionamentos, ao serem respondidos, definem os métodos a serem utilizados: Avaliar para quê? Avaliação de quê? Para quem? (SANTA CATARINA, 2008).

\section{MARCO CONCEITUAL E ESTRATÉGIAS DE PESQUISA}

A temática específica de avaliação em saúde possui vasto referencial teórico e ampla diversidade de abordagens metodológicas e marcos conceituais para descrevê-la. O mais atual desses modelos de avaliação, e que parece ter maior possibilidade de apreender a subjetividade e a complexidade do processo avaliativo, é a chamada Avaliação de Quarta Geração.

Desenvolvida no final da década de 1990, por Guba e Lincoln, a Avaliação de Quarta Geração teve como objetivo superar as limitações das outras três gerações de avaliação e é conduzida por pressupostos metodológicos do modelo construtivista, no qual adota um modelo de avaliação responsiva. Nesse novo paradigma, as preocupações, reivindicações e questões dos grupos de interesse são os eixos norteadores de todo o processo, agregando, nesse cenário, a participação dos sujeitos em todas as etapas de elaboração da pesquisa (KANTORSKI ET AL., 2009).

Apesar dos avanços na construção de um saber no campo da avaliação, qualquer método para apreender a realidade apresenta limitações. As principais dizem respeito ao longo tempo que elas podem requerer, e às indisponibilidades das informações (MARCONI; LAKATOS, 2004). Ressalta-se assim que os modelos "poderão ser utilizados concomitantemente, um apoiando o outro na busca de respostas e mudanças geradas pela avaliação" (COSTA ET AL., 2008, P.719).

A análise da produção brasileira na temática da avaliação em saúde no contexto da ESF, resumida no quadro 2, mostrou uma diversidade de marcos conceituais adotados. Revelou, ainda, um aporte teórico em pesquisadores consagrados internacionalmente, 
como Avedis Donabedian e Bárbara Starfield, e novas propostas, como a do uso de ICSAP enquanto avaliação indireta da Atenção Básica (AB).

A avaliação em saúde, principalmente aquela relacionada à qualidade dos serviços de saúde, tem como um dos seus expoentes Avedis Donabedian. A partir de seus trabalhos, a avaliação dos serviços passou a envolver a dimensão do desempenho técnico e do relacionamento pessoal, e considerar a qualidade como aspecto fundamental do processo avaliativo (REIS ET AL., 1990). De acordo com Donabedian (1990), a qualidade dos serviços é sustentada por sete pilares: eficácia, efetividade, eficiência, otimização, aceitabilidade, legitimidade e equidade.

No modelo de avaliação da qualidade, Donabedian aborda o tripé estrutura, processo e resultado. A estrutura analisa os recursos em termos como força de trabalho, condições físicas, equipamentos e instalações, e demais aspectos que possam garantir a adequação aos padrões e critérios estabelecidos. O processo procura analisar se as atividades e as tarefas são executadas da maneira prevista e se lançam mão de técnicas apropriadas. Por fim, o resultado foca sua análise na capacidade do serviço de atingir seus objetivos, repercutindo na melhoria das condições sanitárias da população (SERAPIONI; LOPES; GURGEL, 2013).

Em um dos estudos elencados nesta pesquisa, a avaliação de implantação lançou mão de dois marcos conceituais (CAMARGO JR., 2008). Um desses marcos envolve a perspectiva avaliativa de Contradiopoulos e Champagne (1997), que exige que se estabeleça um julgamento de adequação e integridade do funcionamento de um programa, associado aos princípios da qualidade de avaliação de Kessner, Kalk e Singer (1992), que consideram os indicadores de cobertura, da qualidade das estruturas e dos processos envolvidos como balizadores desse julgamento.

A condição de ser um sistema universal de saúde, organizado a partir das bases da APS, faz com que a avaliação dos atributos desta seja um método frequentemente utilizado por pesquisadores brasileiros. Tais atributos a serem avaliados consistem de: primeiro contato, longitudinalidade, integralidade, coordenação, centralização familiar e orientação comunitária (STARFIELD, 2002). A literatura registra instrumentos de avaliação da APS com ênfase na aferição da presença e da extensão dos atributos essenciais e derivados da mesma, destacando-se, no cenário brasileiro, o PCATool (LEÃO; CALDEIRA; OLIVEIRA, 2011). A partir do PCATool-Brasil, as avaliações dos atributos da APS se operacionalizam e ganham rigor científico ao permitirem identificar aspectos de estrutura e processo de serviços que exigem reafirmação ou reformulação na busca da qualidade, tanto no planejamento como para a execução das ações de APS (BRASIL, 2010).

Em um dos estudos analisados nesta pesquisa, Cardoso e Vieira-da-Silva (2012) optaram por trabalhar com o conceito de avaliação da cobertura como balizador da avaliação da ESF, considerando que esta avaliação da cobertura refere-se à disponibilidade e à distribuição dos serviços de saúde. Nesse sentido, as autoras se apropriaram da diferenciação de Soberón (1988), de cobertura potencial (relacionada à capacidade e à possibilidade da oferta) e cobertura real (que busca estimar a população que de fato utilizou os serviços). Tal análise possui utilidade analítica ainda pouco explorada no contexto das avaliações da ESF.

Entre os tipos de avaliação das ações e serviços sanitários, os indicadores da atividade hospitalar também representam uma medida de efetividade da APS. E as Condições Sensíveis à Atenção Primária (CSAP) representam, de acordo com Alfradique et al. (2009, P. 1.337), "um conjunto de problemas de saúde para os quais a efetiva ação da atenção primária diminuiria o risco de internações". Ainda segundo essas autoras, o marco conceitual para as CSAP assume que, para algumas condições de saúde, a $\mathrm{AB}$ oportuna 
e de boa qualidade pode evitar ou reduzir o risco de internações.

As hospitalizações por causas evitáveis servem de instrumento de avaliação e monitoramento da efetividade da atenção primária. No Brasil, o seu uso tem se dado de maneira crescente após o Ministério da Saúde ter lançado, no ano de 2008, a Lista Brasileira de Condições Sensíveis à Atenção Primária (NEDEL ET AL., 2010).

A Política de Avaliação da Coordenação de Acompanhamento e Avaliação da Atenção Básica foi um dos textos base para a condução de estudos avaliativos no cenário da ESF. De acordo com essa política, a avaliação em saúde teria, como propósito fundamental, que dar suporte à tomada de decisão, subsidiando a identificação de problemas e a reorientação de ações e serviços, além de avaliar a incorporação de novas práticas sanitárias e mensurar o impacto das ações implementadas pelos serviços e programas que se destinam à saúde das populações (BRASIL, 2005).

Os inquéritos de saúde também são considerados fontes de informação para o procedimento de uma avaliação de desempenho dos sistemas de saúde (CHESTER ET AL., 1996). Nesse sentido, no ano de 2001, a Organização Mundial da Saúde propôs a elaboração da Pesquisa Mundial em Saúde como parte de um projeto de avaliação de desempenho (STARFIELD, 2001), que, no Brasil, encontrou espaço na pesquisa de Szwarcwald, Mendonça e Andrade (2006), revelando a pluralidade de possibilidades de tipologias avaliativas.

Por fim, destaca-se uma nova proposta de avaliação de Tanaka (2011), utilizando a abordagem sistêmica de Donabedian, com a modificação de que a avaliação desse serviço deveria iniciar pelo componente 'processo', visando identificar a adequação da oferta e das relações entre os distintos procedimentos desse nível de atenção. Faz parte da abordagem a avaliação da rede de atenção, tendo como foco e imagem-objetivo a $\mathrm{AB}$ como porta de entrada da rede. Apresenta uma concepção de rede mais dinâmica e propõe a utilização, na avaliação, do método misto, englobando a abordagem quantitativa baseada nos bancos de dados existentes no Departamento de Informática do Sistema Único de Saúde (Datasus) e complementada pela abordagem qualitativa. Nesse desenho metodológico, o autor aponta que será possível a identificação das variáveis de funcionamento e da organização da Atenção Básica e da rede de serviços, possibilitando direcionar a tomada de decisão para a melhoria de qualidade da $\mathrm{AB}$.

\section{Considerações finais}

A partir do momento em que a avaliação em saúde no Brasil ganhou maior repercussão, através dos propósitos e resultados dos estudos aplicados pelo corpo de pesquisadores na área da avaliação, implicados com a saúde coletiva e com a APS, passou-se a socializar o conhecimento através das publicações científicas, a fim de construir o respaldo para as metodologias e estratégias de pesquisas utilizadas, bem como para as tomadas de decisões na gestão do SUS.

O presente estudo de análise da produção científica possibilitou ampliar os conhecimentos sobre as abordagens metodológicas e conceituais das pesquisas em avaliação no contexto da ESF, reforçando o posicionamento estratégico desta na atenção primária.

A análise bibliográfica também revela como a avaliação permite uma produção científica diversificada em conceitos e métodos. Nesse ponto, a existência de estudos de caracteres quantitativos, qualitativos e mistos, além de marcos conceituais diversos, tais como abordagens ligadas aos modelos propostos por Starfield e Donabedian, e a utilização das Condições Sensíveis à Atenção Primária como marcador de qualidade/acesso à $\mathrm{AB}$, revelam esse cenário de pluralismo metodológico. Tais abordagens metodológicas e os marcos conceituais utilizados nos estudos avaliativos com a ESF respeitam as várias visões de políticas públicas de saúde, bem como o olhar 
sobre a operacionalização dos sistemas de saúde, e convergem para o potencial que a ESF tem, de fortalecedora e organizadora da atenção primária.

Além disso, um olhar crítico sobre os autores, as regiões das instituições de esnino superior de origem dos estudos e os periódicos demonstram um predomínio das regiões Sul e Sudeste, sugerindo que as demais regiões do Brasil ainda convivem com as limitações impostas pelas diferenças regionais e sociais para o desenvolvimento de ensino e pesquisa no País.

Os achados desta pesquisa apontam a necessidade da incorporação de múltiplas modalidades de avaliação no monitoramento dos programas e das políticas criadas pelo Ministério da Saúde. Nesse sentido, a institucionalização da avaliação no setor saúde, no Brasil, não deve perder de vista sua pluralidade conceitual e metodológica, a fim de garantir um processo avaliativo amplo e provocador de mudanças.

$\mathrm{O}$ acúmulo de conhecimentos produzido pelos estudos avaliados indica a necessidade de continuarmos a avançar na área de avaliação e de ampliarmos o escopo de instituições e pesquisadores com competências e habilidades técnicas para o desenvolvimento de estudos avaliativos em todo o País.

\section{Referências}

ALFRADIQUE, M. E. et al. Internações por condições sensíveis à atenção primária: a construção da lista brasileira como ferramenta para medir o desempenho do sistema de saúde (Projeto ICSAP - Brasil). Cad. Saúde Pública, Rio de Janeiro, v. 25, n. 6, p. 1337-1349, 2009.

BRASIL. Secretaria de Atenção à Saúde. Avaliação na atenção básica em saúde: caminhos da institucionalização. Brasília, DF: Ministério da Saúde, 2005.

Ministério da Saúde. Secretaria de Atenção à

Saúde. Manual do instrumento de avaliação da atenção primária à saúde: Primary Care Assessment ToolBrasil. Brasília: Ministério da Saúde, 2010. 80 p.

CARDOSO, M. O., VIEIRA-DA-SILVA, L.M. Avaliação da cobertura da atenção básica à saúde em Salvador, Bahia, Brasil (2000 a 2007). Cad. Saúde Pública, Rio de Janeiro, v. 28, n.7, p. 1273-1284, 2012.

CAMARGO, J. R. et al. Avaliação da atenção básica pela ótica político-institucional e da organização da atenção com ênfase na integralidade. Cad. Saúde Pública [internet], v. 24, supl. 1, 2008. Disponível em: <http://www. scielo.br/scielo.php?script=sci_arttext\&pid=S0102-311X2008001300011>. Acesso em: 10 out. 2014.

CHESTER, L. G. C. et al. Morbidade referida e utilização de serviços de saúde em localidades urbanas brasileiras: metodologia. Rev. Saúde Publ., São Paulo, v. 30, n. 2, p. 153-60, 1996.
CONTANDRIOPOULOS, A. P.; CHAMPAGNE, F. A avaliação na área da saúde: conceitos e métodos. In: HARTZ, Z. M. A., (Org:). Avaliação em saúde: dos modelos conceituais à prática na análise da implantação de programas. Rio de Janeiro: Editora Fiocruz; 1997. 29-47 p.

COSTA, G. D. et al. Avaliação em saúde: reflexões inscritas no paradigma sanitário. Physis: Revista Saúde Coletiva, Rio de Janeiro, v. 18, n. 4, p. 705-26, 2008.

DONABEDIAN, A. The seven pillars of quality. Arch Pathol Lab Med, Chicago, v. 114, n. 11, p. 1115-8, 1990.

FACHIN, O. Fundamentos de metodologia. São Paulo: Saraiva, 2006.

GOMES, F. M; SILVA, M. G. C. Programa Saúde da Família como estratégia de atenção primária: uma realidade em Juazeiro do Norte. Ciencia \& Saúde Coletiva, Rio de Janeiro, v. 16, supl. 1, 2011. Disponível em:

$<$ http://www.scielo.br/scielo.php?script=sci_arttext\&pi $\mathrm{d}=$ S1413-81232011000700021 >. Acesso em: 10 out. 2014.

HENRIQUE, F.; CALVO, M. C. M. Avaliação do Programa Saúde da Família nos municípios do Estado de Santa Catarina, Brasil. Cad. Saúde Pública, Rio de Janeiro, v. 24, n. 4, p. 809-19, 2008.

KANTORSKI, L. P. et al. Avaliação de quarta geração: contribuições metodológicas para avaliação de serviços de saúde mental. Interface (Botucatu), Botucatu, v. 13, 
n. 31, out./dez. 2009.

KESSNER, D. M.; KALK, C. E.; SINGER, J. Evaluación de la calidad de la salud por el método de los procesos trazadores. In: Organización Panamericana de la Salud, organizador. Investigaciones sobre servicios de la salud. Brasília: Organización Panamericana de la Salud, 1992. p. 555-63.

LEÃO, C. D. A.; CALDEIRA, A. P.; OLIVEIRA, M. M. C. Atributos da atenção primária na assistência à saúde da criança: avaliação dos cuidadores. Rev. Bras. Saude Mater. Infantil, Recife, v. 11, n. 3, set. 2011.

LENTSCK, M. H; KLUTHCOVSKY, A. C. G. C; KLUTHCOVSKY, F. A. Avaliação do Programa Saúde da Família: uma revisão. Ciência \&t Saúde Coletiva, Rio de Janeiro, v. 15, supl. 3, p. 3455-3466, nov. 2010.

\section{MARCONI, M. A.; LAKATOS, E. M. Metodologia}

Científica. 4. ed. São Paulo: Atlas, 2004.

MINAYO, M. C. S.; ASSIS, S. G.; SOUZA, E. R. Avaliação por triangulação de métodos. Rio de Janeiro: Editora FIOCRUZ, 2005.

MINAYO, M. C. S. (Org.). Pesquisa Social: teoria, método e criatividade. 21. ed. Petrópolis: Vozes, 1993.

MOURA, E. R. F.; HOLANDA, J. R. F.; RODRIGUES, M. S. P. Avaliação da assistência pré-natal oferecida em uma microrregião de saúde do Ceará, Brasil. Cad. Saúde Pública, Rio de Janeiro, v. 19, n. 6, p. 1791-9, nov./dez. 2003.

NEDEL, F. B. et al. Características da atenção básica associada ao risco de internar por condições sensíveis à atenção primária: revisão sistemática de literatura. Epidemiologia e Serviços de Saúde, Brasília DF, v. 19, n. 1, p. 61-75, 2010.

REIS, E. J. F. B. et al. Avaliação da qualidade dos serviços de saúde: notas bibliográficas. Cad. Saúde Pública, Rio de Janeiro v. 6, n. 1, 1990.

SANTA CATARINA. Secretaria de Estado da Saúde, Superintendência de Planejamento e Gestão, Diretoria de Educação Permanente em Saúde. Capacitação para o sistema de planejamento do SUS em Santa Catarina: análise e avaliação em saúde [internet]. Florianópolis: Escola de Saúde Pública Professor Mestre Osvaldo de Oliveira Maciel, 2008. Disponível em : <https://www.google.com. $\mathrm{br} /$ url? $\mathrm{sa}=\mathrm{t} \& \mathrm{rct}=\mathrm{j} \& \mathrm{q}=\&$ esrc $=\mathrm{s} \&$ source $=$ web $\& \mathrm{~cd}=1 \& \mathrm{cad}=\mathrm{r}$ ja\&uact=8\&ved=0CB4QFjAA\&url=http\%3A\%2F\%2Fpor talses.saude.sc.gov.br\%2Findex.php\%3Foption\%3Dcom docman\%26task\%3Ddoc_download\%26gid\%3D2293\%26 Itemid\%3D82\&ei=SgEoVaakCIe-ggSoy4GgCA\&usg=AFQ jCNFZqGYvPT36NbKob3L7qQjVZoJBfQ\&sig2=U4ME4 kfRM5U7ErSQSH3utw>. Acesso em: 10 mar. 2014

SERAPIONI, M.; LOPES, C. M. N.; SILVA, M. G. C. Avaliação em Saúde. In: ROUQUYROL, M. Z.; SILVA, M. G. C. Epidemiologia \&t Saúde. 7. ed. Rio de Janeiro: Medbook, 2013. 533-557 p.

SOBERÓN, G. La extensión de cobertura de los servicios de salud. Gac Méd Méx. Distrito Federal, 1988; n. 124, p. 163-75.

STARFIELD, B. Atenção primária: equilíbrio entre necessidades de saúde, serviços e tecnologia. Brasília, DF: UNESCO, Ministério da Saúde, 2002. 72p.

STARFIELD, B. Improving equity in health: a research agenda. Int J Health Serv, New York, v. 31, n. 3, p. 54566, 2001.

\section{SZWARCWALD, C. L.; MENDONÇA, M. H. M.;} ANDRADE, C. L. T. Indicadores de atenção básica em quatro municípios do Estado do Rio de Janeiro, 2005: resultados de inquérito domiciliar de base populacional. Ciência \&t saúde coletiva, Rio de Janeiro, v. 11, n. 3, set. 2006.

TANAKA, O. Y. Avaliação da atenção básica em saúde: uma nova proposta. Saúde e sociedade, São Paulo, v. 20, n. 4, dez. 2011.

TURATO, E. R. Métodos qualitativos e quantitativos na área de saúde: definições, diferenças e seus objetos de pesquisa. Revista de Saúde Pública, São Paulo, n. 39, v. 3, p. 507-14.

\footnotetext{
Recebido para publicação em abril de 2014

Versão final em novembro de 2014

Conflito de interesses: inexistente

Suporte financeiro: não houve
} 